\title{
Communication
}

\section{Is it possible to improve communication around radiotherapy delivery: A randomized study to assess the efficacy of team training?}

\author{
Aurore Liénard ${ }^{\mathrm{a}, \mathrm{b}, 1}$, France Delevallez ${ }^{\mathrm{a}, 1}$, Darius Razavi ${ }^{\mathrm{a}, \mathrm{b}}$, Anne-Sophie Gibon ${ }^{\mathrm{a}}$, Yves Libert ${ }^{\mathrm{a}, \mathrm{b}}$, \\ Nicole Delvaux ${ }^{\mathrm{a}, \mathrm{b}, \mathrm{c}}$, Serge Marchal ${ }^{\mathrm{b}, \mathrm{c}, \mathrm{d}}$, Anne-Marie Etienne ${ }^{\mathrm{e}}$, Isabelle Bragard ${ }^{\mathrm{e}}$, Christine Reynaert ${ }^{\mathrm{f}, \mathrm{g}}$, \\ Jean-Louis Slachmuylder ${ }^{\mathrm{d}}$, Jean-François Durieux ${ }^{\mathrm{d}}$, Christine Farvacques ${ }^{\mathrm{d}}$, Pierre Scalliet ${ }^{\mathrm{g}}$, \\ Paul Van Houtte ${ }^{\mathrm{b}, \mathrm{h}}$, Philippe Coucke ${ }^{\mathrm{i}}$, Isabelle Merckaert ${ }^{\mathrm{a}, \mathrm{b}, *}$
}

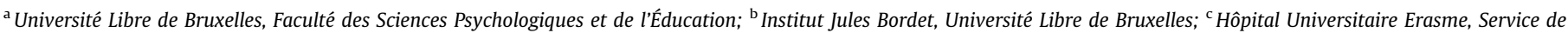

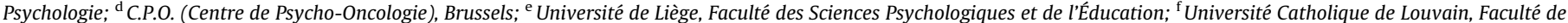

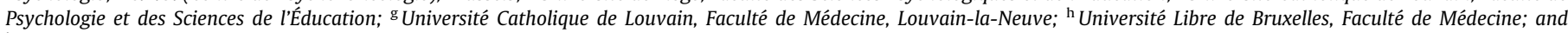
${ }^{i}$ Université de Liège, Faculté de Médecine, Belgium

\section{A R T I C L E I N F O}

\section{Article history:}

Received 18 November 2015

Received in revised form 15 March 2016

Accepted 20 March 2016

Available online 9 April 2016

\section{Keywords:}

Radiotherapy

Communication skills training

Team

Cancer

\begin{abstract}
A B S T R A C T
Background and purpose: The aim of this study was to assess the efficacy of a 38-h communication skills training program designed for multidisciplinary radiotherapy teams.

Materials and methods: Four radiotherapy teams were randomly assigned to a training program or to a waiting list. Assessments were scheduled at baseline (T1) and then after the training was completed or four months later (T2), respectively. Communication around radiotherapy delivery was assessed based on audio recordings of the first and last radiotherapy sessions in order to assess team members' communication skills and the expression of concerns by breast cancer patients (analyzed with content analysis software LaComm).

Results: 198 radiotherapy sessions were recorded. During the first radiotherapy sessions, members of the trained teams exhibited more assessment skills $(p=0.048)$, provided more setting information $(p<0.001)$, and used more social words $(p=0.019)$ compared to the members of the untrained teams. During the last radiotherapy session, members of the trained teams used more assessment skills $(p=0.004)$ and patients interacting with members of the trained teams expressed more sadness words $(p=0.023)$.

Conclusion: Training of multidisciplinary teams has the potential to transfer skills that affect the short exchanges that take place around radiotherapy delivery.
\end{abstract}

(C) 2016 Elsevier Ireland Ltd. All rights reserved. Radiotherapy and Oncology 119 (2016) 361-367
Over the last few decades, several randomized controlled trials have assessed the efficacy of communication skills training programs [1]. These communication skills training programs mainly focused on training individuals, and evaluated dyadic exchanges in the context of two-person interviews [1-4]. Interestingly, specific care contexts, such as radiotherapy delivery, have rarely been studied.

Communication around radiotherapy delivery is challenging for several reasons. First, radiotherapy is a highly technical treatment; second, treatment delivery is invisible; and third, side-effects often have a delayed onset or can fluctuate throughout treatment [5]. As

\footnotetext{
* Corresponding author at: Université Libre de Bruxelles, Av. F. Roosevelt, 50 - CP 191, B-1050 Bruxelles, Belgium.

E-mail address: Isabelle.Merckaert@ulb.ac.be (I. Merckaert).

1 A.L. and F.D. contributed equally to this work and should be considered co-first authors.
}

a result, patients often express numerous concerns regarding radiotherapy treatment and its side effects [6-10]. Furthermore, many patients experience feelings of anxiety at the start of their treatment $[6,11]$. Communication in the radiotherapy context involves the transmission of complex information, and regular assessment of the patients' physical state and of his or her information and supportive needs. Indeed, studies have shown that patients are mainly dissatisfied due to a perceived lack of information [12-14].

Communication exchanges with patients in radiotherapy have been found to primarily occur near the treatment machine [15] or when patients move from the waiting area to the treatment room [16]. These exchanges are often short and they occur in uncomfortable contexts. In addition, radiotherapy treatments involve various professionals who need to be aware of their different roles and are not consistently matched with the same patients over 
many weeks of treatment. Therefore, continuity of care for patients requires that information transmission occurs between professional colleagues. Optimal communication regarding radiotherapy delivery requires that radiotherapy team members possess a repertoire of skills that allow them to communicate with both patients and their colleagues. However, it is important to note that healthcare professionals involved with radiotherapy treatment typically deal with a heavy workload and have many time constraints, and this may prevent them from providing high quality psychosocial care [16]. Therefore, professionals need to be trained to master the skills needed to balance both the physical and emotional needs of a patient during treatments, and this training is seldom provided [17].

To our knowledge, only two communication skills training programs have focused on this particular care context [18,19]. In the first study, the training of radiation oncologists resulted in an increase in patients' participation in the initial radiation oncology consultations [18]. Second, in previously published results of the present study, completion of the training program allowed the team members to transfer their learned communication skills within the context of radiotherapy planning appointments and patients' satisfaction with their care was improved [19].

It was hypothesized that the learning and transfer of communication skills that are relevant in clinical practice would be promoted by a team training. Positive team attitudes from colleagues toward the skills could also further optimize the transfer [20]. In the present study, the first and last sessions were chosen for assessing the transfer of communication skills since these sessions are often highly technical and very short, similar to all of the sessions of treatment. However, the first and last sessions represent significant encounters for the patient. For example, at the first session of treatment, patients often experience fears regarding their treatment $[9,21]$, clinically relevant anxiety $[6,11]$, they express numerous treatment-related information needs [7], and it is often their first contact with the machine that will deliver their treatment. During the first session, team members have an opportunity to create a relationship with the patient, to transmit information about what will happen and how, and to evaluate the patient's emotional state. In contrast, the last session of treatment represents a final opportunity for patients to have daily contact with the medical team. Patients may express mixed feelings that reflect relief as a result of their treatment being completed, anxiety due to uncertainty about the future and long-term side effects, or they present depressive affects [6,7].

Therefore, the aim of the present study was to assess the impact of a 38-h communication skills training program on the transfer of learned skills in radiotherapy sessions using a randomized controlled study design. In particular, communication that occurred during the first and last radiotherapy sessions was assessed. It was hypothesized that the training program would lead to an increase in the transmission of treatment-related and setting information at the first treatment session. Then, at the last session, the professionals in the trained teams would potentially use directive and checking questions more frequently to verify each patient's information needs [7]. In both sessions, it was anticipated that the professionals in the trained teams would use more openassessment and support skills and would be more receptive to patients' expression of concerns than the members of the untrained teams.

\section{Materials and methods}

\section{Study design and assessment procedures}

Four multidisciplinary radiotherapy teams were randomly allocated prior to the first assessment time to either a 38-h training program (training group) or a waiting-list (waiting-list group). To be included, team members needed to be able to speak French and needed to be willing to participate in the training program and its assessment procedures. Among the 217 recruited members, a total of 96 team members took part in the training program: $68 \%$ $(n=65)$ of these subjects were members of the teams allocated to the training group and $32 \%(n=31)$ were members of the teams that were allocated to the waiting-list group. All team members agreed to participate in the assessment procedure. Assessments for the training group were scheduled following randomization (T1) and again after the training program (T2). Assessments for the waiting-list group were scheduled at T1 and 4 months after T1 (T2) (Fig. 1). Two different cohorts of breast cancer patients were recruited at the four months interval: one cohort participated in the $\mathrm{T} 1$ assessment and one cohort participated in the T2 assessment. Audio recordings were made for the first and last radiotherapy sessions for each patient in both cohorts. These recordings were started upon the arrival of the patients in the radiotherapy unit and ended when the patients left the unit.

\section{Communication skills training program}

The 38-h communication skills training program included two modules: a patient-oriented communication skills training module and a subsequent team-resource-oriented communication skills training module. Sessions were scheduled over a 4-month period, which allowed team members to practice their newly acquired skills and were organized into small groups (5-9 participants). Training was learner-centered, skills-focused, and practiceoriented. It included cognitive, behavioral, and modeling components $[22,23]$.

The 16-h patient-oriented communication skills training module included five sessions. The first 4-h session included the entire radiotherapy team and focused on presenting information regarding patients' distress during radiotherapy and conducting practical exercises involving communication in oncology. The other four 3-h sessions were organized into small mono-disciplinary groups and were designed to improve the team members' abilities to communicate with patients according to their own professional roles.

The 22-h team-resource-oriented communication skills training module included seven sessions. The six 3-h sessions were organized in small interdisciplinary groups, including at least one team member from each discipline, and included 1-h of information summarizing different forms of collaboration, and 17-h of roleplaying exercises. These sessions were designed to improve each team member's ability to communicate with both patients and colleagues. At the end of the training program, the entire radiotherapy team took part in a 4-h session which provided a summary of the previous sessions, it assessed the participants' satisfaction, and it facilitated a discussion about the training program. A more detailed description of this training program has previously been published [17].

\section{Radiotherapy sessions}

The transfer of acquired communication skills was assessed in the first and last radiotherapy sessions of cancer patients undergoing radiotherapy for breast cancer. To participate in this study, the following criteria had to be met: being able to speak French, being at least 18 years old, diagnosed for the first time with nonmetastatic breast cancer, receiving a first radiotherapy treatment, and being free of cognitive impairment. There were two different cohorts of patients that were recruited, one at $\mathrm{T} 1$ and one at $\mathrm{T} 2$. The patients in both cohorts provided written informed consent, they completed a demographics questionnaire before their radiotherapy planning session, and they completed a Hospital Anxiety 

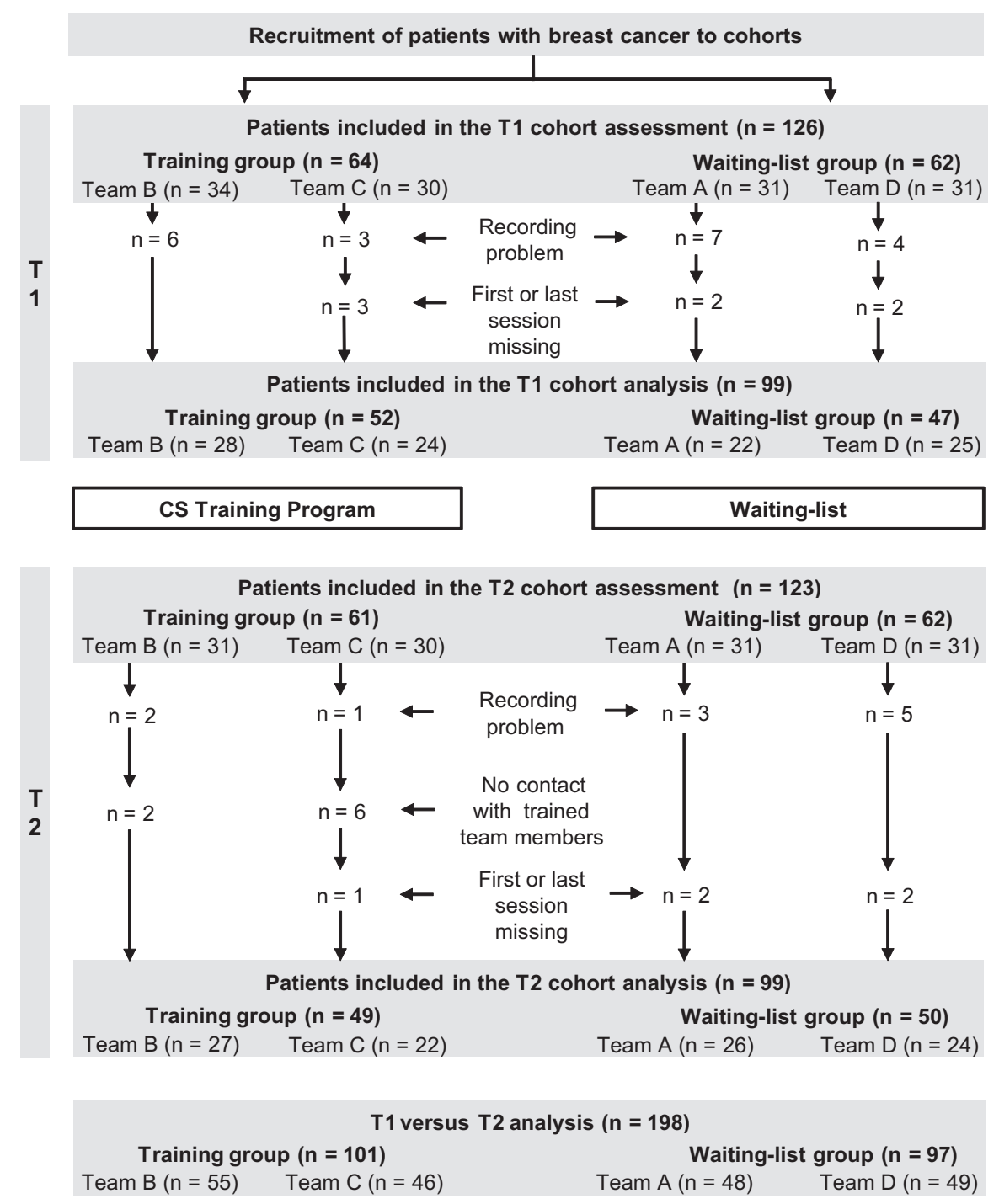

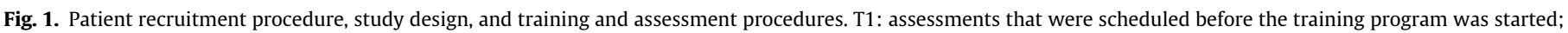
T2: assessments that were conducted 4 months after the first assessment. CS: communication skills; RT: radiotherapy.

and Depression Scale before the first and last radiotherapy sessions in order to be able to compare the cohorts.

The radiotherapy session audiotapes included any interactions that the patients had with any team member on the day of their session. The majority of these interactions were formal and they took place at the reception desk and during radiotherapy sessions. The other interactions were informal and took place in the waiting room and corridors.

The patients had to have communicated with at least one trained team member during the radiotherapy sessions to be considered for inclusion in the data analysis. All of the exchanges that occurred between the included patients and any of the members of the teams were included in our analysis. Interactions that occurred between professionals were not taken into account in order to most closely approximate the patients' experience.

\section{Communication content analysis}

Audiotapes of the radiotherapy sessions were transcribed and analyzed by a French communication content analysis software, LaComm (Centre de Psycho-Oncologie). This software analyzes verbal medical communication utterance by utterance, and identifies turns of speech, and the type and content of utterances. The explanation of this software has been previously published [24]. Utterances were categorized into three main types: assessment, support, and information. Regarding utterance content, four categories of dictionaries were constructed: radiotherapy, medical, emotional (sadness and anxiety), and social (eAppendix [Supplement]). The content of these dictionaries were built based on empirical knowledge derived from actual and simulated patient consultations in oncology [2]. Use of the LaComm software was based on its sensitivity $[25,26]$ and its avoidance of inter-rater reliability problems. A validation study $[25,26]$ showed that the sensitivity to change of the LaComm software was similar to that described for the Cancer Research Campaign Workshop Evaluation Manual (CRCWEM) [27]. Due to the number of speakers and the turns of speech included in the audio recordings analyzed, the allocation category was supervised to increase the efficacy of the LaComm software.

\section{Questionnaires}

Sociodemographic data

Patients provided demographic information. Physicians provided data regarding patients' disease and treatment characteristics. 
Hospital Anxiety and Depression Scale

The Hospital Anxiety and Depression Scale (HADS) [28] is a 14item, four-point self-report instrument that assesses anxiety and depression in physically ill subjects. The HADS has previously been validated in a sample of cancer inpatients [29]. The use of the total score is recommended to assess psychological distress [29].

\section{Statistical analyses}

LaComm provided counts of utterance types and content. These data were considered dependent variables and group-by-time effects were tested with generalized estimating Poisson regression models. The models tested time effects, group allocation effects, and group-by-time effects using the training group at baseline and the waiting-list group as the reference group. Training effects were controlled for the number of team members' turns of speech.

Statistical analyses of patients' sociodemographic data included a comparative analysis of both groups (e.g., patients' interactions with team members of the training group $v s$. with team members of the waiting-list group) using the nonparametric tests, $\chi^{2}$ and Kruskal-Wallis, as appropriate. Group-by-time differences in patients' anxiety, depression, and distress HADS scores during the course of radiotherapy treatment were examined using a two-way analysis of variance (ANOVA) as appropriate when the data are not normally distributed, but variances are homogeneous (as determined with Levene's test) [30]. An a priori significance level of $\alpha=0.05$ was applied for all tests. Analyses were performed with SPSS software (version 22.0 for Windows; IBM Corp., Armonk, NY, USA).

\section{Results}

\section{Composition of the teams and socioprofessional data}

Among the 65 members of the trained teams, 10 were secretaries, 30 were nurses, 14 were physicians, and 11 were physicists. The mean age of the trained team members was $39 \pm 10.6$ years, $71 \%$ were females, and $92 \%$ lived with a partner or children. In addition, $66 \%$ of the trained team members worked full-time and had $12 \pm 9.9$ years of experience in oncology and $10 \pm 9.5$ years of experience with their present team. Within the last year, seven team members attended a patient-oriented training and twelve attended a team-oriented training.

Among the 31 members of the untrained teams, 3 were secretaries, 19 were nurses, and 9 were physicians. No physicists participated in the communication skills training program in the untrained team. The mean age of the untrained team members was $41 \pm 8.9$ years, $71 \%$ were females, and $74 \%$ lived with a partner or children. In addition, $80 \%$ of the untrained team members worked full-time and had $13 \pm 8.9$ years of experience in oncology and $9 \pm 8.8$ years of experience with their present team. Within the last year, two team members had attended a patient-oriented training and six had attended a team-oriented training.

There were no statistically significant differences at baseline between both teams, except regarding their marital status. A greater number of the untrained team members were single $(p=0.015)$.

\section{Patient recruitment and sociodemographic data}

Of the 249 patients who accepted and participated in our study, 51 were excluded for the following reasons: recording problems $(n=31)$, the patients were not undergoing their first or last session of treatment $(n=12)$, or the patients had a lack of contact with trained team members $(n=8)$. Of the 198 patients who were included in the analysis, 101 patients encountered members of the trained teams ( 52 at $\mathrm{T} 1$ and 49 at T2), and 97 patients encountered members of the untrained teams ( 47 at T1 and 50 at T2). Similar numbers of patients for each team were recruited (Fig. 1).

At T2, the patients who encountered professionals from the trained teams communicated on average with three team members in the first session and four team members in the last session. All patients communicated with at least one trained team member. More precisely, $74 \%$ of patients in the first session and $80 \%$ of patients in the last session communicated with at least two trained team members; $31 \%$ of patients in the first session and $39 \%$ of patients in the last session communicated with at least three trained team members; and $10 \%$ of patients in the first session and $10 \%$ of patients in the last session communicated with at least four trained team members.

\section{Patient characteristics}

The only significant group-by-time differences detected between the patients of both groups concerned occupational status $(p=0.001)$ and number of lumpectomies $(p=0.015)$. In the waiting-list group, fewer patients had undergone a lumpectomy at $\mathrm{T} 1$ and fewer patients at $\mathrm{T} 2$ worked at least part-time (eAppendix [Supplement]). There were also no significant group-by-time differences between the patients of both groups in terms of anxiety, depression, and distress levels at both sessions (eAppendix [Supplement]).

Training effects on team members' utterances during radiotherapy sessions

Generalized estimating equation for Poisson regression analyses (GEE) showed no significant group-by-time effects on the number of team members' turns of speech in both sessions. Concerning the first radiotherapy session, generalized estimating equation analyses showed significant group-by-time effects on the utterance-type counts made by team members (Table 1 ). At T2 compared with baseline, analyses showed a significant increase in the rate of open and open directive questions (relative rate $(\mathrm{RR})=8.71 ; p=0.048$ ) for members of the trained teams compared with members of the untrained teams (used as the reference group). At T2 compared with baseline, analyses showed a significant increase in the rate of setting information ( $R R=1.44$; $p<0.001)$ and in the count of social word ( $R R=1.40 ; p=0.019)$ for members of the trained teams compared with members of the untrained teams.

Concerning the last radiotherapy session, analyses showed significant group-by-time effects on the utterance-type counts made by team members (Table 2). At T2 compared with baseline, analyses showed a significant increase in the rate of checking questions $(R R=2.13 ; p=0.010)$, directive questions $(R R=1.76 ; p=0.029)$, and total questions ( $R R=1.88 ; p=0.004$ ) for members of the trained teams compared with members of the untrained teams. In contrast, no changes were observed in the last radiotherapy session contents.

\section{Training effects on patients' utterances during radiotherapy sessions}

GEE for Poisson regression analyses showed no significant group-by-time effect on the number of patients' turns of speech in both sessions. Concerning the first radiotherapy session, GEE showed no changes on the utterance-type counts and in the radiotherapy session contents, compared with baseline, for patients interacting with members of the trained teams compared to patients interacting with members of the untrained teams (Table 1). 
Table 1

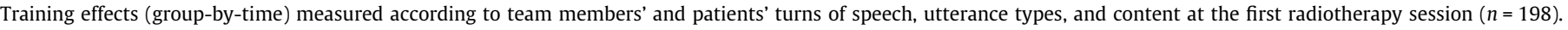

\begin{tabular}{|c|c|c|c|c|c|c|c|c|c|c|c|}
\hline & \multicolumn{4}{|c|}{ Training group $(n=101)$} & \multicolumn{4}{|c|}{ Waiting-list group ( $n=97)$} & \multirow{2}{*}{\multicolumn{3}{|c|}{$\begin{array}{c}\text { Generalized estimating equation } \\
\text { Training effects }\end{array}$}} \\
\hline & \multicolumn{2}{|c|}{$\mathrm{T} 1(n=52)$} & \multicolumn{2}{|c|}{$\mathrm{T} 2(n=49)$} & \multicolumn{2}{|c|}{$\mathrm{T} 1(n=47)$} & \multicolumn{2}{|c|}{$\mathrm{T} 2(n=50)$} & & & \\
\hline & Mean & SD & Mean & SD & Mean & SD & Mean & SD & $\mathrm{RR}$ & $95 \% \mathrm{CI}$ & $p$ \\
\hline \multicolumn{12}{|l|}{ Turns of speech } \\
\hline Patients & 91.3 & 50.9 & 85.2 & 53.2 & 116.8 & 42.5 & 95.3 & 51.0 & 1.14 & $0.86-1.53$ & 0.366 \\
\hline Team members & 109.1 & 63.8 & 102.9 & 62.4 & 149.9 & 61.4 & 113.7 & 58.2 & 1.24 & $0.93-1.67$ & 0.146 \\
\hline Physicians & 0.9 & 2.7 & 0.8 & 3.1 & 1.2 & 5.6 & 2.8 & 15.5 & 0.37 & $0.03-4.11$ & 0.423 \\
\hline Nurses & 104.0 & 60.1 & 99.0 & 60.2 & 145.9 & 60.0 & 107.7 & 53.7 & 1.29 & $0.96-1.72$ & 0.088 \\
\hline Secretaries & 4.1 & 9.2 & 3.1 & 7.6 & 2.7 & 5.6 & 3.1 & 10.8 & 0.65 & $0.16-2.72$ & 0.555 \\
\hline \multicolumn{12}{|l|}{ Team members' utterances } \\
\hline \multicolumn{12}{|l|}{ Types } \\
\hline \multicolumn{12}{|l|}{ Assessment } \\
\hline Open and open directive questions & 0.02 & 0.1 & 0.2 & 0.5 & 0.5 & 0.8 & 0.4 & 0.9 & 8.71 & $1.02-74.76$ & 0.048 \\
\hline Leading questions & 0.02 & 0.1 & 0.02 & 0.1 & 0.02 & 0.2 & 0.1 & 0.3 & 0.23 & $0.01-8.02$ & 0.415 \\
\hline Checking questions & 2.9 & 2.5 & 2.4 & 2.1 & 3.0 & 2.0 & 2.6 & 2.2 & 0.79 & $0.54-1.14$ & 0.203 \\
\hline Directive questions & 2.8 & 2.7 & 2.7 & 2.7 & 3.3 & 2.4 & 2.8 & 2.4 & 0.96 & $0.64-1.44$ & 0.836 \\
\hline Total $^{*}$ & 5.8 & 4.7 & 5.4 & 4.4 & 6.9 & 3.7 & 5.9 & 4.2 & 0.89 & $0.65-1.22$ & 0.472 \\
\hline \multicolumn{12}{|l|}{ Support } \\
\hline Acknowledgment & 20.5 & 15.2 & 19.9 & 11.6 & 27.4 & 12.0 & 25.7 & 13.8 & 0.85 & $0.70-1.03$ & 0.094 \\
\hline Empathy $^{*}$ & 0.1 & 0.3 & 0.2 & 0.5 & 0.2 & 0.4 & 0.1 & 0.4 & 2.25 & $0.49-10.31$ & 0.295 \\
\hline Reassurance ${ }^{*}$ & 1.2 & 1.3 & 1.2 & 1.7 & 2.4 & 2.8 & 1.2 & 1.5 & 1.34 & $0.73-2.44$ & 0.343 \\
\hline Total & 21.8 & 15.8 & 21.3 & 12.7 & 30.0 & 13.5 & 27.0 & 14.2 & 0.88 & $0.73-1.06$ & 0.191 \\
\hline \multicolumn{12}{|l|}{ Information } \\
\hline Setting information & 9.8 & 4.4 & 12.1 & 5.8 & 13.5 & 5.7 & 10.2 & 5.4 & 1.44 & $1.18-1.75$ & $<0.001$ \\
\hline Negotiation & 8.2 & 6.6 & 7.3 & 4.6 & 9.7 & 6.9 & 7.1 & 6.0 & 1.07 & $0.73-1.57$ & 0.740 \\
\hline \multicolumn{12}{|l|}{ Contents } \\
\hline Medical words & 30.1 & 18.1 & 29.8 & 16.8 & 36.0 & 20.9 & 25.3 & 18.6 & 1.24 & $0.91-1.69$ & 0.177 \\
\hline Radiotherapy words & 30.4 & 16.4 & 30.6 & 17.7 & 37.3 & 16.4 & 27.4 & 14.6 & 1.15 & $0.96-1.39$ & 0.131 \\
\hline Anxiety words & 2.2 & 2.4 & 1.9 & 2.3 & 3.6 & 3.5 & 2.3 & 2.1 & 1.09 & $0.68-1.74$ & 0.726 \\
\hline Sadness words ${ }^{*}$ & 0.5 & 1.1 & 0.5 & 0.8 & 0.6 & 0.8 & 0.3 & 0.7 & 1.67 & $0.67-4.13$ & 0.269 \\
\hline Social words & 14.9 & 8.1 & 18.2 & 10.0 & 23.9 & 10.9 & 19.0 & 11.8 & 1.40 & $1.06-1.84$ & 0.019 \\
\hline \multicolumn{12}{|l|}{ Patients' utterances } \\
\hline \multicolumn{12}{|l|}{ Types } \\
\hline \multicolumn{12}{|l|}{ Assessment } \\
\hline Open questions* & 0.4 & 0.7 & 0.2 & 0.6 & 0.6 & 1.0 & 0.3 & 0.7 & 0.85 & $0.28-2.56$ & 0.777 \\
\hline Directive questions ${ }^{*}$ & 1.7 & 2.1 & 1.5 & 1.8 & 1.3 & 1.3 & 1.6 & 1.5 & 0.60 & $0.37-1.05$ & 0.075 \\
\hline Total $^{*}$ & 5.1 & 4.8 & 4.1 & 4.4 & 4.5 & 3.7 & 3.8 & 3.5 & 0.86 & $0.57-1.30$ & 0.478 \\
\hline \multicolumn{12}{|l|}{ Contents } \\
\hline Medical words & 8.4 & 10.7 & 6.7 & 6.5 & 11.2 & 8.6 & 6.8 & 7.4 & 1.41 & $0.92-2.16$ & 0.119 \\
\hline Radiotherapy words & 8.1 & 6.9 & 8.2 & 6.8 & 11.3 & 8.5 & 7.9 & 7.6 & 1.29 & $0.90-1.85$ & 0.171 \\
\hline Anxiety words & 1.0 & 1.7 & 1.2 & 1.7 & 0.9 & 1.2 & 0.6 & 0.9 & 1.68 & $0.77-3.68$ & 0.196 \\
\hline Sadness words ${ }^{*}$ & 0.3 & 0.7 & 0.3 & 0.8 & 0.1 & 0.3 & 0.2 & 0.4 & 0.50 & $0.12-2.06$ & 0.340 \\
\hline Social words & 7.9 & 6.0 & 8.6 & 7.1 & 10.3 & 9.0 & 8.7 & 8.5 & 1.16 & $0.80-1.68$ & 0.429 \\
\hline
\end{tabular}

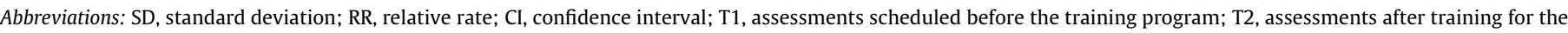
training group and 4 months after T1 for the waiting-list group.

Estimated RR based on generalized linear Poisson regression models adjusted for the number of turns of speech (except for the turns of speech variable).

Negative binomial distribution.

Concerning the last radiotherapy session, GEE showed no significant changes in the utterance-type counts but showed a significant increase in the count of sadness word $(\mathrm{RR}=5.86 ; p=0.023)$ for patients interacting with members of the trained teams compared to patients interacting with members of the untrained teams (Table 2).

\section{Discussion}

The results of the present study demonstrate that a 38-h training program enabled the transfer of learned communication skills within communication exchanges that occurred around radiotherapy delivery. As expected, higher rates of open and open directive questions, and of setting information were used by members of the trained teams compared to members of the untrained teams during the first radiotherapy session. Higher rates of social words use were also observed in the first radiotherapy sessions. The increased rate of setting information discussed may indicate that the members of the trained teams paid greater attention to the explanation of events and with whom they would occur. The higher rate of social words expression by the members of the trained teams may also indicate that attempts were made to build a relationship with the patients. Regarding the last radiotherapy session, higher rates of checking and directive questions were observed for members of the trained teams compared with members of the untrained teams. These results suggest that the members of the trained teams were more attentive to having a good understanding of the transmitted information and were also more attentive to the comfort of the patients. It was also observed that the patients interacting with members of the trained teams used more sadness words than the patients interacting with members of the untrained teams. This result appears to indicate that the patients were provided with the opportunity to express their 
Table 2

Training effects (group-by-time) measured based on team members' and patients' turns of speech, utterance types, and content at the last radiotherapy session ( $n=198$ ).

\begin{tabular}{|c|c|c|c|c|c|c|c|c|c|c|c|}
\hline & \multicolumn{4}{|c|}{ Training group $(n=101)$} & \multicolumn{4}{|c|}{ Waiting-list group $(n=97)$} & \multirow{2}{*}{\multicolumn{3}{|c|}{$\begin{array}{c}\text { Generalized estimating equation } \\
\text { Training effects }\end{array}$}} \\
\hline & \multicolumn{2}{|c|}{$\mathrm{T} 1(n=52)$} & \multicolumn{2}{|c|}{$\mathrm{T} 2(n=49)$} & \multicolumn{2}{|c|}{$\mathrm{T} 1(n=47)$} & \multicolumn{2}{|c|}{ T2 $(n=50)$} & & & \\
\hline & Mean & $\mathrm{SD}$ & Mean & SD & Mean & SD & Mean & $\mathrm{SD}$ & $\mathrm{RR}$ & $95 \% \mathrm{CI}$ & $p$ \\
\hline \multicolumn{12}{|l|}{ Turns of speech } \\
\hline Patients & 64.5 & 37.8 & 56.8 & 27.8 & 57.5 & 24.2 & 65.4 & 37.7 & 0.77 & $0.58-1.03$ & 0.080 \\
\hline Team members & 73.0 & 42.7 & 65.1 & 33.4 & 71.9 & 34.9 & 65.4 & 51.0 & 0.80 & $0.59-1.09$ & 0.162 \\
\hline Physicians* & 0.8 & 3.1 & 0.8 & 3.3 & 0.3 & 1.5 & 0.1 & 0.6 & 1.89 & $0.14-24.83$ & 0.629 \\
\hline Nurses & 59.8 & 33.8 & 55.0 & 32.1 & 70.8 & 35.1 & 78.4 & 49.2 & 0.83 & $0.61-1.14$ & 0.247 \\
\hline Secretaries ${ }^{*}$ & 12.4 & 22.1 & 9.3 & 12.6 & 0.8 & 3.0 & 1.3 & 3.6 & 0.48 & $0.12-1.97$ & 0.307 \\
\hline \multicolumn{12}{|l|}{ Team members' utterances } \\
\hline \multicolumn{12}{|l|}{ Types } \\
\hline \multicolumn{12}{|l|}{ Assessment } \\
\hline Open and open directive questions* & 0.1 & 0.3 & 0.2 & 0.4 & 0.3 & 0.4 & 0.3 & 0.5 & 1.45 & $0.42-5.06$ & 0.558 \\
\hline Leading questions" & 0.1 & 0.3 & 0.0 & 0.2 & - & - & 0.0 & 0.1 & - & - & - \\
\hline Checking questions & 1.4 & 1.2 & 2.3 & 2.3 & 1.6 & 1.9 & 1.5 & 1.8 & 2.13 & $1.19-3.79$ & 0.010 \\
\hline Directive questions & 1.6 & 1.6 & 2.2 & 1.7 & 1.4 & 1.3 & 1.3 & 1.7 & 1.76 & $1.06-2.91$ & 0.029 \\
\hline Total & 3.1 & 2.3 & 4.7 & 3.4 & 3.3 & 2.7 & 3.1 & 3.2 & 1.88 & $1.23-2.88$ & 0.004 \\
\hline \multicolumn{12}{|l|}{ Support } \\
\hline Acknowledgment & 14.1 & 9.7 & 12.1 & 8.9 & 12.3 & 8.7 & 14.3 & 12.5 & 1.05 & $0.82-1.36$ & 0.683 \\
\hline Empathy $^{*}$ & 0.1 & 0.5 & 0.0 & 0.1 & 0.2 & 0.5 & 0.0 & 0.3 & 0.96 & $0.04-22.38$ & 0.979 \\
\hline Reassurance ${ }^{*}$ & 0.3 & 0.7 & 0.2 & 0.6 & 0.2 & 0.4 & 0.4 & 0.9 & 0.43 & $0.11-1.70$ & 0.230 \\
\hline Total & 14.6 & 10.0 & 12.4 & 8.9 & 12.7 & 8.8 & 14.8 & 12.5 & 1.04 & $0.81-1.33$ & 0.761 \\
\hline \multicolumn{12}{|l|}{ Information } \\
\hline Setting information & 7.9 & 4.3 & 8.0 & 3.8 & 7.8 & 4.1 & 7.0 & 4.1 & 1.25 & $0.95-1.63$ & 0.109 \\
\hline Negotiation & 2.3 & 2.2 & 2.5 & 1.9 & 2.2 & 1.6 & 2.9 & 2.7 & 0.93 & $0.59-1.45$ & 0.744 \\
\hline \multicolumn{12}{|l|}{ Contents } \\
\hline Medical words & 8.1 & 9.1 & 7.6 & 5.8 & 8.8 & 5.7 & 9.2 & 8.1 & 1.09 & $0.72-1.64$ & 0.689 \\
\hline Radiotherapy words & 6.8 & 6.8 & 5.2 & 3.9 & 6.0 & 4.8 & 7.3 & 5.0 & 0.80 & $0.55-1.18$ & 0.257 \\
\hline Anxiety words & 0.8 & 1.5 & 0.3 & 0.8 & 0.6 & 1.0 & 0.5 & 1.3 & 0.63 & $0.18-2.25$ & 0.479 \\
\hline Sadness words & 0.1 & 0.4 & 0.3 & 0.6 & 0.3 & 0.6 & 0.2 & 0.6 & 3.60 & $0.91-14.27$ & 0.068 \\
\hline Social words & 9.9 & 8.3 & 8.3 & 5.6 & 9.7 & 7.3 & 9.4 & 6.8 & 1.08 & $0.76-1.52$ & 0.672 \\
\hline \multicolumn{12}{|l|}{ Patients' utterances } \\
\hline \multicolumn{12}{|l|}{ Types } \\
\hline \multicolumn{12}{|l|}{ Assessment } \\
\hline Open questions* & 0.1 & 0.5 & 0.1 & 0.4 & 0.3 & 0.5 & 0.1 & 0.3 & 4.57 & $0.92-22.64$ & 0.062 \\
\hline Directive questions ${ }^{*}$ & 0.8 & 1.2 & 1.1 & 1.5 & 0.6 & 0.8 & 0.6 & 1.0 & 1.87 & $0.87-4.02$ & 0.106 \\
\hline Total $^{*}$ & 2.4 & 2.8 & 2.4 & 2.3 & 2.0 & 2.1 & 1.9 & 1.8 & 1.53 & $0.92-2.56$ & 0.102 \\
\hline \multicolumn{12}{|l|}{ Contents } \\
\hline Medical words & 6.6 & 10.5 & 4.6 & 5.2 & 4.4 & 3.8 & 5.3 & 7.0 & 1.18 & $0.76-1.84$ & 0.456 \\
\hline Radiotherapy words ${ }^{*}$ & 4.7 & 6.3 & 2.4 & 2.6 & 2.9 & 2.3 & 3.7 & 3.1 & 0.66 & $0.41-1.08$ & 0.101 \\
\hline Anxiety words & 0.7 & 1.5 & 0.5 & 1.0 & 0.4 & 0.7 & 0.5 & 1.2 & 0.66 & $0.19-2.23$ & 0.501 \\
\hline Sadness words ${ }^{*}$ & 0.3 & 0.6 & 0.4 & 0.8 & 0.2 & 0.4 & 0.1 & 0.3 & 5.86 & $1.28-26.81$ & 0.023 \\
\hline Social words & 9.2 & 10.7 & 7.4 & 6.3 & 7.4 & 5.8 & 7.8 & 8.8 & 1.47 & $0.95-2.29$ & 0.087 \\
\hline
\end{tabular}

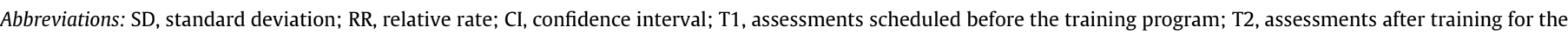
training group and 4 months after $\mathrm{T} 1$ for the waiting-list group.

Estimated RR based on generalized linear Poisson regression models adjusted for the number of turns of speech (except for the turns of speech variable).

Negative binomial distribution.

feelings. Overall, the communication skills exhibited by the members of the trained teams in the first and the last radiotherapy sessions somewhat differed, yet they were consistent with the specific goals of the two sessions.

An unexpected and important finding was that patient support did not appear to differ between the two groups. Moreover, some communication strategies (such as open and open-directive questions) were rarely used even after training. Most of the communication exchanges were mainly centered on medical and radiotherapy contents. The changes observed seem however to be meaningful for patients as patients who have encountered the members of the trained teams have been shown previously to be more satisfied with nurses interventions [19]. There is therefore still a need for improvement. It is possible that the organization of consolidation workshops would be helpful, and the type of training conducted could include a coaching component whereby the participants first observe interactions around the treatment machine, and then test various strategies that could be used in these context-specific exchanges.

There were limitations associated with the present study. First, only four radiotherapy teams were included, and these teams had different histories, leadership types, and organizational backgrounds. The participation rate for the members of each team also differed between the groups, and a higher participation rate was observed for the teams that were allocated to the training arm of the study [17]. Second, the LaComm software assessed the impact of training on verbal communication, although it does not take into account different communication contexts and sequences. Third, the analyses performed did not completely control for potential confounders of the clustering effects between teams. 
The present results also demonstrate that further research is needed to assess the impact of training in the context of radiotherapy delivery, specifically regarding the context and organization of care that is provided. In the present study, the aim of the training performed was not to change the service organization that was already established but in order to maintain and improve communication skills, the advantages of organizational support should be considered $[3,31]$.

In conclusion, the type of training program employed in the present study was found to be efficient and it facilitated the transfer of learned communication skills to the workplace even during the short exchanges that took place around the highly technical context of radiotherapy delivery. Moreover, the results of the present study demonstrate that this type of training program is acceptable (regular participation in training sessions can be achieved) and feasible (if the training is adapted to the time constraints of a radiotherapy unit).

\section{Funding source role}

Sponsors of this study did not contribute to the study design, data collection, data analysis, data interpretation, or the preparation, review, or approval of the final manuscript.

\section{Conflict of interest statement}

The authors have no financial or personal relationships with people or organizations that inappropriately influenced this work. The principal investigator had full access to all of the data for this study and assumes full responsibility for data integrity and analysis accuracy.

\section{Acknowledgments}

The "Fonds National de la Recherche Scientifique - Section

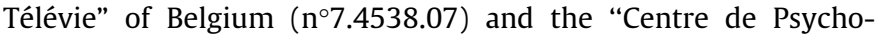
Oncologie" of Brussels provided support for this research program. The authors would like to thank the following radiotherapy teams that participated in this study: the "Institut Jules Bordet - ULB" in Brussels (Professor Paul Van Houtte); the "Cliniques universitaires Saint-Luc" in Brussels (Professor Pierre Scalliet); the "Clinique Saint-Elisabeth" in Namur (Professor Emile Salamon); and the "Centre Hospitalier Universitaire" of Liège (Professor Philippe Coucke).

\section{Appendix A. Supplementary data}

Supplementary data associated with this article can be found, in the online version, at http://dx.doi.org/10.1016/j.radonc.2016.03. 016.

\section{References}

[1] Moore PM et al. Communication skills training for healthcare professionals working with people who have cancer. Cochrane Database Syst Rev 2013;3 CD003751.

[2] Razavi D et al. How to optimize physicians' communication skills in cancer care: results of a randomized study assessing the usefulness of post training consolidation workshops. J Clin Oncol 2003;21:3141-9.
[3] Heaven C et al. Transfer of communication skills training from workshop to workplace: the impact of clinical supervision. Patient Educ Couns 2006;60:313-25.

[4] Lienard A et al. Transfer of communication skills to the workplace during clinical rounds: impact of a program for residents. PLoS ONE 2010;5: e12426.

[5] Jereczek-Fossa BA et al. Radiotherapy-related fatigue. Crit Rev Oncol Hematol 2002;41:317-25.

[6] Stiegelis HE et al. Psychological functioning in cancer patients treated with radiotherapy. Patient Educ Couns 2004;52:131-41.

[7] Halkett GK et al. Meeting breast cancer patients' information needs during radiotherapy: what can we do to improve the information and support that is currently provided? Eur J Cancer Care (Engl) 2010;19:538-47.

[8] Halkett GK et al. Information needs and preferences of women as they proceed through radiotherapy for breast cancer. Patient Educ Couns 2011;86:396-404.

[9] Long LE. Being informed: undergoing radiation therapy. Cancer Nurs $2001 ; 24: 463-8$.

[10] Douma KL et al. Do patients' information needs decrease over the course of radiotherapy? Support Care Cancer 2012:20:2167-76.

[11] Lewis $\mathrm{F}$ et al. Anxiety and its time courses during radiotherapy for nonmetastatic breast cancer: a longitudinal study. Radiother Oncol 2014; $111: 276-80$.

[12] Zissiadis Y et al. Patient satisfaction in radiation oncology. Australas Radiol 2006;50:455-62.

[13] Sandoval GA et al. Factors that influence cancer patients' overall perceptions of the quality of care. Int J Qual Health Care 2006;18:266-74.

[14] Nguyen TVF et al. Determinants of patient satisfaction in ambulatory oncology: a cross sectional study based on the OUT-PATSAT35 questionnaire. BMC Cancer 2011;11:526.

[15] Halkett GK, Kristjanson LJ. Patients' perspectives on the role of radiation therapists. Patient Educ Couns 2007;69:76-83.

[16] Merchant S et al. Time, space and technology in radiotherapy departments: how do these factors impact on patients' experiences of radiotherapy? Eur. J. Cancer Care 2015:n/a-a.

[17] Gibon AS et al. Is it possible to improve radiotherapy team members' communication skills? A randomized study assessing the efficacy of a 38hour communication skills training program. Radiother Oncol 2013;109:170-7.

[18] Timmermans LM et al. Enhancing patient participation by training radiation oncologists. Patient Educ Couns 2006:63:55-63.

[19] Merckaert I et al. Transfer of communication skills to the workplace: impact of a 38-hour communication skills training program designed for radiotherapy teams. J Clin Oncol 2015.

[20] Williams C et al. The doctor-patient relationship: from undergraduate assumptions to pre-registration reality. Med Educ 2001;35:743-7.

[21] Halkett GKB et al. 'If we get too close to your bones they'll go brittle': women's initial fears about radiotherapy for early breast cancer. Psycho-Oncology 2008; 17:877-84.

[22] Stiefel $\mathrm{F}$ et al. Communication skills training in oncology: a position paper based on a consensus meeting among European experts in 2009. Ann Oncol 2010;21:204-7.

[23] Merckaert I et al. Communication skills training in cancer care: where are we and where are we going? Curr Opin Oncol 2005;17:319-30.

[24] Lienard A et al. Is it possible to improve residents breaking bad news skills? A randomised study assessing the efficacy of a communication skills training program. Br J Cancer 2010;103:171-7.

[25] Gibon A-S et al. Developement of the Lacomm, a French medical communication analysis software: A study assessing its sensitivity to change. Psychooncology 2010;19:133-4.

[26] Gibon A-S et al. Development of the LACOMM, A French Medical Communication Analysis Software: A Study Assessing its Sensitivity to Change, 2015 [Unpublished article].

[27] Booth K, Maguire P. Development of a Rating System to Assess Interaction Between Cancer Patients and Health Professionals, 1991.

[28] Zigmond AS, Snaith RP. The hospital anxiety and depression scale. Acta Psychiatr Scand 1983;67:361-70.

[29] Razavi D et al. Screening for adjustment disorders and major depressive disorders in cancer in-patients. Br J Psychiatry 1990;156:79-83.

[30] Larson MG. Analysis of Variance. Circulation 2008;117:115-21.

[31] Uitterhoeve RJ et al. The effect of communication skills training on patient outcomes in cancer care: a systematic review of the literature. Eur. J. Cancer Care 2010;19:442-57. 